\title{
Effect of hydrophilic or hydrophobic interactions on the self-assembly behavior and micro-morphology of a collagen mimetic peptide
}

\author{
Xiaomin Luo ${ }^{1,2^{*}}$, Qianqian Huo ${ }^{1,2}$, Xinhua Liu ${ }^{3^{*}}$, Chi Zheng ${ }^{1,2}$ and Ying Liu ${ }^{1,2}$
}

\begin{abstract}
Peptide self-assembles with bionic properties have been widely utilized for bioactive drugs and biomedical materials. Collagen mimetic peptide (CMP) gains more attention due to its unique advantages in biosecurity and function. Unfortunately, the self-assembly mechanism of CMP, particularly the effect of intermolecular forces on its self-assembly behavior and morphology, is still unrecognized. Herein, the hydrophilic glycidol (GCD) and hydrophobic Y-glycidyl ether oxypropyl trimethoxysilane $(G L H)$ were grafted onto the side chains of CMP through the ring-opening reaction (GCD/CMP, GLH/CMP). Subsequently, the effects of hydrophilic and hydrophobic interactions on the self-assembly behavior and morphology of CMP were further studied. The results substantiated that the GCD/CMP and GLH/CMP self-assembly followed "nucleation-growth" mechanism, and the supererogatory hydrophilic and hydrophobic groups prolonged the nucleation and growth time of CMP self-assembly. Noted that the hydrophilic interaction had stronger driving effects than hydrophobic interaction on the self-assembly of CMP. The GCD/CMP and GLH/CMP self-assembles exhibited fibrous 3D network and microsphere morphology, respectively. Furthermore, the GLH/CMP self-assembles had better resistance to degradation. Consequently, the microtopography and degradation properties of CMP self-assembles could be controlled by the hydrophilic and hydrophobic interactions between CMP, which would further provide a way for subsequent purposeful design of biomedical materials.
\end{abstract}

Keywords: Collagen mimetic peptide, Hydrophilic interaction and hydrophobic interaction, Self-assembly, Morphology

\section{Introduction}

Polypeptide is recognized as one of the most promising natural organic polymer in the field of biomedical materials due to its analogous nature to ECM [1-3]. However, collagen extracted from animal tissues has potential disadvantages such as the introduction of

\footnotetext{
* Correspondence: luoxiaomin@sust.edu.cn; liuxinhua@sust.edu.cn

${ }^{1}$ College of Bioresources Chemical and Materials Engineering, Shaanxi

University of Science \& Technology, Wei Yang District, Xi'an 710021, Shaanxi,

China

${ }^{3}$ Institute of Biomass \& Functional Materials, Shaanxi University of Science \&

Technology, Weiyang District, Xi'an 710021, Shaanxi, China

Full list of author information is available at the end of the article
}

foreign pathogenic genes and immune rejection [4]. At present, at least $5 \%$ of the population still has immune rejection reactions to collagen products [5-8], seriously affecting its biomedical applications. However, collagen mimetic peptide (CMP) is a kind of recombinant polypeptide with high biological activity, high biocompatibility and many functionalities, which is synthesized through a series of optimized modification processes by using genetic engineering technology to simulate the amino acid sequence of human collagen structurally [912]. Repeated literatures has confirmed that the structure and function of CMP could been precisely bio- 
designed in advance, so that compared to animalderived collagen, CMP has abundant incomparable advantages including magnificent structural controllability, no animal-derived viruses and diverse functions [13-16]. Therefore, the CMP based biomedical materials with high performance and high added value is more in line with the requirements of clinical applications [17].

Polypeptide molecules can self-assemble into more ordered aggregates in nature with bionic structures, diverse functions and wide-ranging applications through non-covalent interactions, which make it more potential in the development of bioactive peptide drugs and biomedical materials [18-23]. Non-covalent bonds such as hydrogen/hydrophobic bonds, van der Waals forces, cation- $\pi$ adsorption and other interaction forces are the key to the self-assembly process [24, 25]. Coincidentally, evidence to date indicates that once polypeptide are biosynthesized in vivo and it would self-assemble at the nano-, micro-, and macro-scales into more advanced polypeptide aggregates in a complex hierarchical way [26]. Hierarchical organization at every structural level of polypeptide materials allows for the intervention and interplay of a series of design features [27]. The selfassembled architectures could not only reinforce the mechanical attributes of tissues and more preferably biomimic the biological characteristics of the native extracellular matrix, but also promote their chemical-physical characteristics, functional features and structural stability. Therefore, the understanding of self-assembly behavior of polypeptide could contribute to regulate the anticipatory properties of the aggregated materials at the molecular level to meet various practical applications $[28,29]$. According to the performance improvement from polypeptide to self-assembled polypeptide aggregates, this enlightens us that the self-assembled CMP aggregates with extremely biomimetic structure of natural polypeptide in tissues might have, more superior comprehensive performances than that of CMP ontology [30-32]. However, the current research on CMP and its assembly system is in the primary research stage in terms of structural complexity, multi-level, functionality, and application, especially the study on its assembly behavior is still blank.

CMP is an amphiphilic biological protein [33]. Similar to animal-derived nature polypeptide, a large number of hydrophilic and hydrophobic amino acids are arranged in the CMP molecular chain [34]. And thus according to the amphiphilic nature of CMP, under different conditions, CMP molecules should suffice to self-assemble to aggregates with different hierarchical secondary structures theoretically (such as $\beta$ sheets and $\alpha$ helices) through weak interactions between non-covalent bonds, which could further fine-tune the micro-topography of self-assembled CMP, including nanotubes, nanofibers, microspheres and micron-level fiber bundles [35]. Actually, the hydrophilic and hydrophobic forces are one of the main driving forces for the self-assembly of amphiphilic peptides, which play an important role in stabilizing the secondary and tertiary conformations [36-38]. Half of the amino acids in the ionic complementary peptide sequence are hydrophobic amino acids, and the extensive hydrophobic interaction between the side chains of the hydrophobic amino acid residues greatly increases the tendency of the ionic complementary peptide molecules to aggregate in an orderly manner, making this type of molecule have a fairly high self-assembly ability. Liao et al. [39] used short peptide amphiphiles (NapPhe-Phe-Lys-pTyr) as a model to study the self-assembly mechanism of nanofibers and found that hydrophobic interaction and ionic interaction were the dominant forces in the self-assembly process. Nevertheless, up to date, the role of hydrophilic and hydrophobic interaction in the self-assembly process of CMP and its influence on the mico-morphology have not been reported [40-42]. The glycidol (GCD) with hydroxyl groups has the magnificent hydrophilic nature and biocompatibility. While Y-glycidyl ether oxypropyl trimethoxysilane (GLH) contains abundant hydrophobic group siloxane, which endows it a certain hydrophobicity [43]. Due to the charge polarization and ring tension in the epoxy group, the epoxy groups has high reactivity and is ready to react with certain groups containing active hydrogen atoms, such as amino, carboxyl, and hydroxyl group, therefore, they are used always graft agents .

Herein, to elaborate the influence of the hydrophilic and hydrophobic interactions on the assembly behavior and performance of CMP, the hydrophilic GCD and hydrophobic GLH were grafted onto the side chains of CMP through the ring-opening reaction (GCD/CMP, GLH/CMP). Then we focus especially on revealing the mechanisms, mico-morphology, kinetics and thermodynamics of the CMP self-assembly process, we expect that this study would provide useful results for understandingthe non-covalent mechanism of CMP assembly and the method of molecular arrangement on nano-scale and macro-scale to form ordered and regular assemblies. Realizing the precise control of the structure morphology and key performance of the assembly aggregates, further utilize and deepen the life body assembly system, and provide inspiration and ideas for the development of new functional assembly systems [44].

\section{Experiments}

\subsection{Materials}

Glycidyl alcohol (GCD) and Y-glycidoxypropyltri methoxysilane (GLH) were purchased from Shanghai Maclean Biochemical Technology Co., Ltd; CMP was purchased from Xi'an Well come Co., Ltd., and its 
amino acid sequence conforms to type I polypeptide. The Gly-XY sequence (shown in Figure S1) has the secondary structure characteristics of a typical polypeptide molecule, with a molecular weight of $38,359 \mathrm{Da}$, and the CMP has no obvious cytotoxicity and has good biocompatibility; other chemical reagents are purchased from Sigma-Aldrich.

\subsection{Preparation of GCD/CMP, GLH/CMP and their homologous assemblies}

\section{(1) Preparation of GCD/CMP, GLH/CMP}

Prepare aqueous solutions of different pH 9.0, 9.5, $10.0,10.5,11.0$, respectively. Weigh a certain amount of CMP and slowly add it to the above solutions, and then introduce a certain amount of GCD, GLH. The molar ratio of amino and epoxy is 1:0, 1:0.5, 1:1, 1:1.5, 1:2, and the mixture is magnetic stirred at $37^{\circ} \mathrm{C}$ for a certain time of $1 \mathrm{~h}, 5 \mathrm{~h}, 9 \mathrm{~h}, 12 \mathrm{~h}, 17 \mathrm{~h}$. A series of GCD/CMP and GLH/CMP solutions were placed in a treated dialysis bag (relative molecular weight cut-off of $10 \mathrm{KD}$ ) for 3 to 4 days. Finally, the GCD/CMP and GLH/CMP were freeze-dried for use.

\section{(2) Preparation of GCD/CMP and GLH/CMP assemblies}

Take $100 \mathrm{~mL}$ of $\mathrm{pH} 7.0$ aqueous solution and slowly add $30 \mathrm{mg}$ of GCD/CMP or GLH/CMP and stir them evenly. After incubating for $600 \mathrm{~min}$ in a biochemical incubator at $37^{\circ} \mathrm{C}$, the GCD/CMP and GLH/CMP assemblies are obtained. Part of the aggregates are freeze-dried for later use. The remaining part is poured into a polytetrafluoroethylene board and placed in an atmospheric oven at $40^{\circ} \mathrm{C}$ for 3 days to obtain bubbles-free, smooth and flat GCD/CMP and GLH/CMP films.

\subsection{Structural characterization of GCD/CMP, GLH/CMP}

The $\mathrm{KBr}$ compression method was used to analyze the structure of the specimens by using Fourier Transform Infrared Spectroscopy (FT-IR) (Spectrum One, PerkinElmer, Inc., Waltham, MA). In a dry environment at room temperature, the $4000 \sim 400 \mathrm{~cm}^{-1}$ wave number bands were scanned 32 times and the average was set to 4 $\mathrm{cm}^{-1}$.

Measure the amino conversion rate by ultravioletvisible-near-infrared spectrophotometer (UV-vis) (Cary 5000, Agilent Technologies) to characterize the degree of hydrophilic or hydrophobic reaction between GCD or GLH and CMP. Determined according to reference [45]. The formula is as follows:

$$
\text { Amino conversation rate } \%=\frac{C_{0}-C_{1}}{C_{0}} \times 100 \%
$$

$C_{0}$ :the content of free amino groups in CMP Film, mg/ $\mathrm{mL}$;

$C_{1}$ :the content of free amino group in sample film, $\mathrm{mg} / \mathrm{mL}$.

\subsection{Self-assembly kinetics of GCD/CMP and GLH/CMP assemblies}

The self-assembly kinetics of GCD/CMP and GLH/CMP composite specimens were monitored by turbidimetric measurement on a Perkin Elmer Lambda 25 UV-vis spectrometer equipped with a thermostat holder. The time course of optical density (turbidity) was measured at $313 \mathrm{~nm}$ every $30 \mathrm{~s}$. Meanwhile, the self-assembly kinetic parameters were characterized quantitatively according to the method of Yan et al. [45].

\subsection{Mico-morphology analysis of GCD/CMP and GLH/CMP assemblies}

The morphology of the GCD/CMP and GLH/CMP assemblies was observed using a scanning electron microscope (SEM). Place the samples on the conductive glue, spray gold, and collect the spectrum at an accelerating voltage of $10 \mathrm{kV}$.

\subsection{Performance characterization of GCD/CMP and GLH/ CMP assemblies}

Cut the films of CMP, GCD/CMP and GLH/CMP assemblies into rectangles, and use video contact angle measuring instrument (OCA-H200, Dataphysics Co., Ltd.) to detect the static contact angle (SCA) of the specimens, and add $5 \mu \mathrm{L}$ of distilled water to the sample surface. The built-in digital camera records the shape of water droplets and then calculates the size of the surface contact angle. Each sample is measured in 3 parallel samples, and is tested three times at three different locations. Cut the films of CMP, GCD/CMP and GLH/CMP assemblies into rectangles, and use a video contact angle meter to detect the dynamic contact angle (DCA) of different films. Add $5 \mu \mathrm{L}$ of distilled water after boiling and cooling to the sample surface, and the built-in digital camera records the shape of water droplets and records the contact angle with the calculated surface. The recording time is $12 \mathrm{~s}$.

Weigh a certain amount $\left(\mathrm{w}_{0}\right)$ of the films of CMP, GCD/CMP and GLH/CMP assemblies into a colorimetric tube containing $5 \mathrm{~mL}$ modified SBF simulated body fluid, and degrade naturally at $37^{\circ} \mathrm{C}$. At predetermined time points $(1,3,5,15$ and 30 days), the specimens were taken out from the medium, and were washed three times with modified SBF simulated body fluid. Then the films were put into a vacuum drying oven at room 
temperature, drying to constant weight $\left(\mathrm{w}_{\mathrm{t}}\right)$. The degradation rates of the specimens were calculated by the following equation:

$$
\text { Degradation rate }(\%)=\frac{\mathrm{w}_{0}-\mathrm{w}_{\mathrm{t}}}{\mathrm{w}_{0}} * 100 \%
$$

\section{Results and discussion}

\subsection{Preparation and structural analysis of GCD/CMP and}

\section{GLH/CMP}

Figure 1 shows a schematic diagram of the hydrophilic and hydrophobic modification reactions between CMP and GCD or GLH. The preparation conditions of GCD/ CMP and GLH/CMP were optimized based on the amino conversion rate (Fig. 1d), which implies that the optimal reaction conditions are 1:1 of the amino group and epoxy group molar ratio, $12 \mathrm{~h}$ of reaction time, $37^{\circ} \mathrm{C}$ of temperature, $\mathrm{pH}$ 10.0. And the optimum amino group conversion rate of GCD/CMP and GLH/CMP could reach $37.9 \%$ and $48.1 \%$, respectively. Figure 1c shows the FT-IR spectra of CMP, GCD, GLH, GCD/ CMP and GLH/CMP. The secondary structure of CMP before and after modification can be directly reflected by the representative amide A, B, I, II and III bands [46]. Figure 1c indicates that the characteristic amide A band of CMP is centered at $3300 \mathrm{~cm}^{-1}$, which is predominantly originated from the $\mathrm{N}-\mathrm{H}$ free stretching vibration peak, and the characteristic absorption peak of amide $\mathrm{B}$ band locates at around at $2920 \mathrm{~cm}^{-1}$, which arises from the asymmetric stretching vibration of $-\mathrm{CH}_{2}$. The amide I band caused by the $\mathrm{C}=\mathrm{O}$ stretching vibration of the amide group in the peptide skeleton could be found at

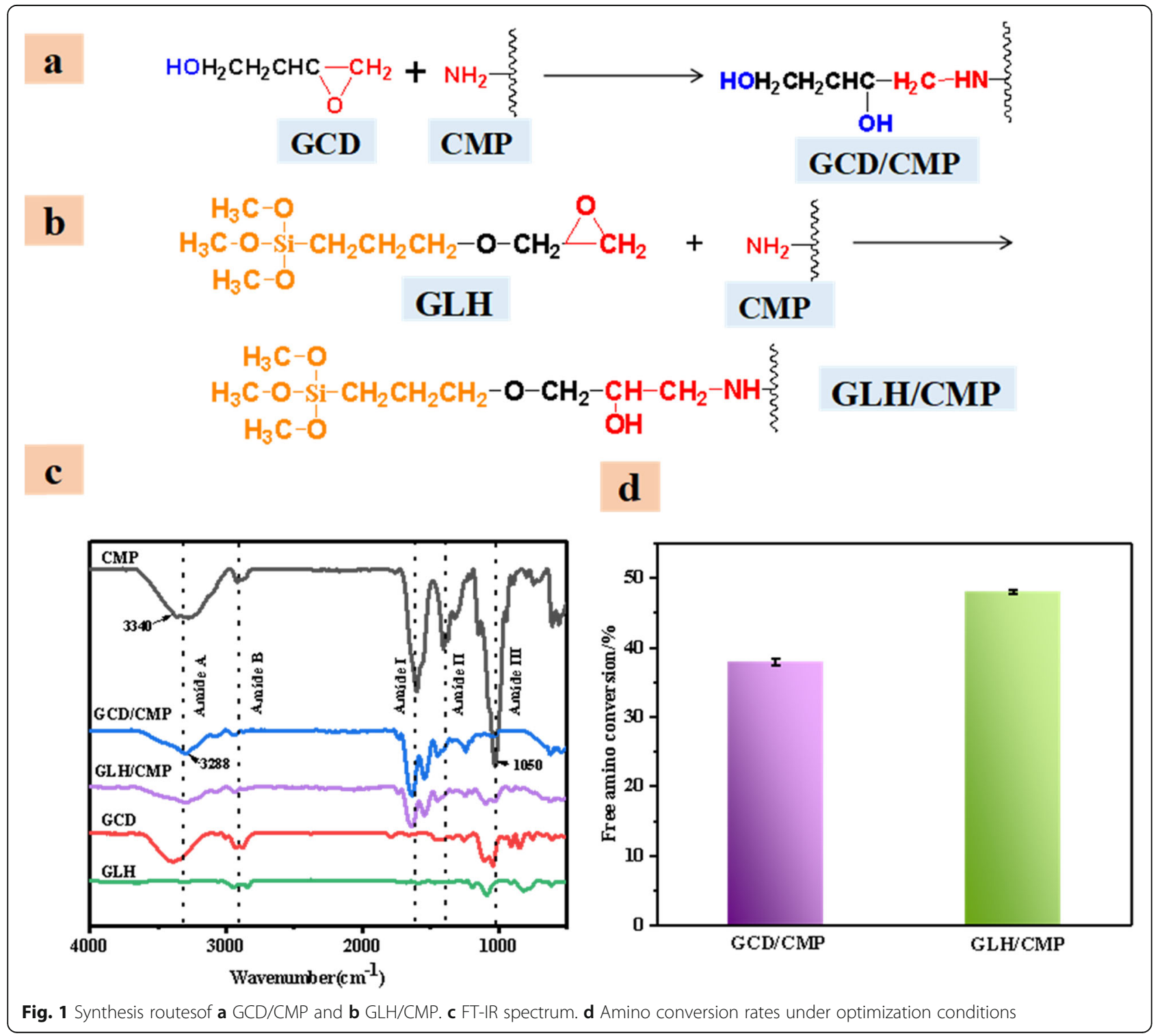


$1627 \mathrm{~cm}^{-1}$, and the amide II band at $1415 \mathrm{~cm}^{-1}$ was attributed to the amide $\mathrm{N}-\mathrm{H}$ bending vibrations and $\mathrm{C}-\mathrm{N}$ stretching vibrations. The two feature amide bands of CMP are closely related to the its secondary structure including $\alpha$-helix, $\beta$-sheet and random coil structure and other components [47]. Besides, The amide III band of CMP could be located at $1290 \mathrm{~cm}^{-1}$, which is assigned to the $\mathrm{C}-\mathrm{N}$ stretching and $\mathrm{N}-\mathrm{H}$ in plane bending from amide linkages, as well as absorptions arising from wagging vibrations from $\mathrm{CH}_{2}$ groups from the glycine backbone and proline side-chains. From Fig. 1c, it can be found that the characteristic absorption peaks of the amide bands of GCD/CMP and GLH/CMP are obviously present, and it is preliminarily confirmed that the GCD and GLH have no negative effects on the basic skeleton structure of CMP.

Further, Fig. 1c indicates the amides I, II and III bands of GCD/CMP and GLH/CMP have a slight decrease in intensity, which substantiates that GCD or GLH might have insignificant negative effects on the hydrogen bonds between CMP molecules [48]. When the N-H group on the GCD/CMP chain participates in the formation of hydrogen bonds, meanwhile, the amide $\mathrm{A}$ band peak of GCD/CMP shifts to a low frequency, indicating that the ring-opening reaction between GCD and CMP may result in the formation of regenerative hydrogen bonds among the amino groups, carboxyl groups, hydroxyl groups and hydroxyl groups on the GCD/CMP chain. Moreover, the disappearance of a strong peak of $\mathrm{GLH} / \mathrm{CMP}$ at $1050 \mathrm{~cm}^{-1}$ may be related to the introduction of $\mathrm{Si}-\mathrm{O}-\mathrm{Si}$ into the siloxane hydrolysis and condensation reaction. Noted that the amide A and B peaks of CMP after GLH or GCD modification with broadening and a slight shift to lower wavenumber to varying degrees could be observed, which manifests that the structural regularity of CMP molecules increases after the introduction of hydrophilic and hydrophobic groups, further hinting the self-assembly of CMP.

\subsection{The self-assembly behavior of CMP, GCD/CMP and GLH/CMP}

\subsubsection{The effect of hydrophilic and hydrophobic groups on} the self-assembly process and morphology of CMP

The self-assembly behavior of CMP, GCD/CMP and GLH/CMP can be analyzed by measuring the turbidity changes. Figure 2 shows the turbidity changes with time in the self-assembly process of CMP, GCD/CMP and GLH/CMP, and the microscopic morphology of the corresponding self-assembles at different magnifications. All the turbidity curves of CMP, GCD/CMP and GLH/CMP exhibit anticipatory " $\mathrm{S}$ " shaped, indicating that the selfassembly process of the GCD/CMP and GLH/CMP are still coincided with a cooperative "nucleation-growth" mechanism,. Based on the shape of the survey curve, the

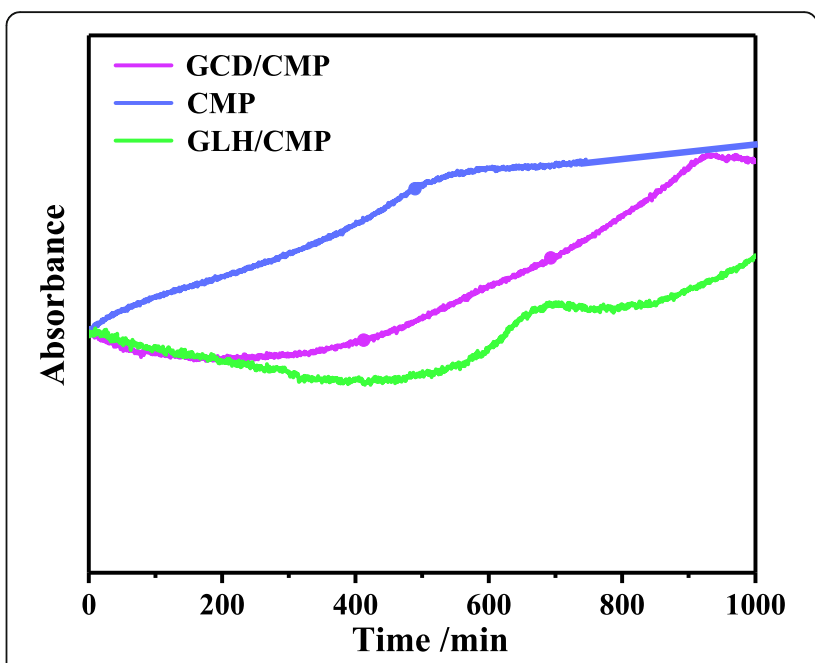

Fig. 2 Turbidity detection diagram

assembly process can be divided into three stages: nucleation stage, growth stage and equilibrium stage. Further, in the nucleation stage, the changes in turbidity are negligible. Subsequently, the nucleation core gradually forms in the growth phase, in which the core gradually grows into aggregates. Finally, the self-assembly process enters the plateau stage where the turbidity tends to reach the equilibrium level. Noted that the completion time and self-assembly rate of each stage of different systems are different, which might be mainly caused by the intermolecular forces between GCD/GLH and CMP. Meanwhile, obvious differences at the initial stages of kinetic curves could be observed between the CMP, GCD/CMP and GLH/CMP, especially for the GLH/ CMP. Due to the nucleation-propagation mechanism of polypeptide, a certain amount of polypeptide molecules associate to form metastable nuclei during the lag phase, upon which further molecules accrete during the growth phase. Figure 2 showed that unlike pure CMP, the absorbance of GCD/CMP and GLH/CMP decreases after the introduction of GCD and GLH, indicating that the introduction of GCD and GLH has significantly negative effects on the formation of CMP nucleation centers to varying degrees. This phenomenon eventually leads to a smaller particle size of GCD/CMP and GLH/CMP assemblies as the size of collagenassemblies is determined by the equilibrium optical density. Reported literatures have also verified that disparate driving forces might have significative effects on the microscopic morphology of the corresponding self-assembles. Particularly, the self-assembly of CMP reaches to the plateau phase after $600 \mathrm{~min}$, where the assembles are mainly composed of vesicles and microtubules, confirmed by Fig. 3b. As for the GCD/CMP, the turbidity reaches equilibrium at 900 min, and in this stage, the GCD/CMP assembles present a fibrous network structure formed by interspersed 


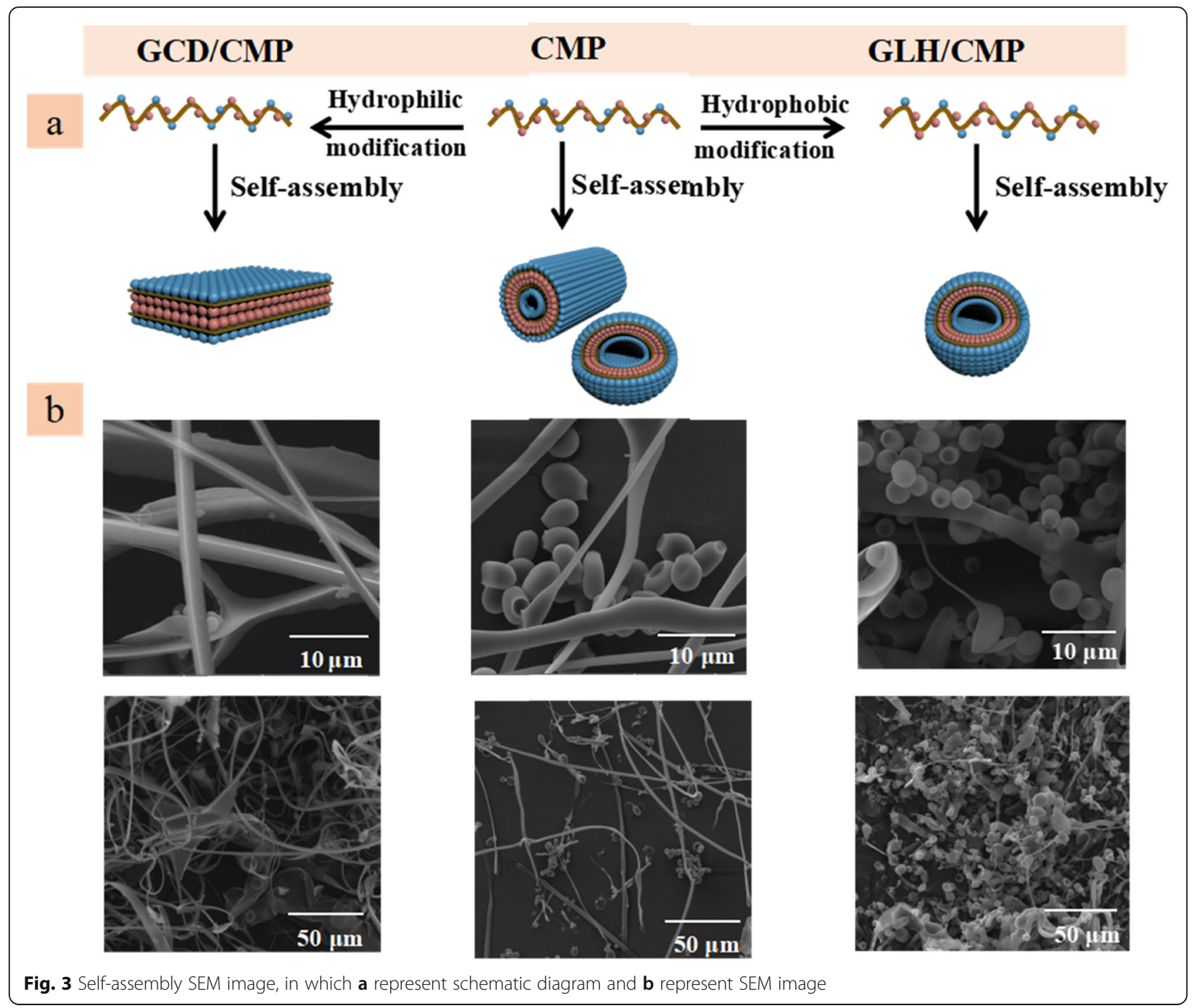

dendritic fibers, which attributes to the reconstructed hydrogen bonds between the hydrophilic groups of GCD and polar groups of CMP. Meanwhile, it takes $800 \mathrm{~min}$ for GLH/CMP self-assembly to reach plateau phase, and the homologous microscopic morphology of GLH/CMP assembles exhibits mainly microspheres and thin ribbons, most of which are microspheres therein, this may be assigned to the hydrophobic bonds between GLH and CMP, which hinders the intermolecular aggregation. All in all, the introduction of hydrophilic groups and hydrophobic groups affects the autonomous nucleation and growth time of CMP molecules simultaneously, leading to the diversified formation of morphological structures in its final aggregates. Moreover, the hydrophilic interaction promotes the assembles to form fibrous aggregates, while the hydrophobic interaction is more conducive to the formation of microspherical appearance.
3.2.2 Kinetics of CMP, GCD/CMP and GLH/CMP selfassembly

The self-assembly kinetics of CMP, GCD/CMP, and GLH/CMP could be characterized by the dynamic changes of absorbance. It was confirmed that the value of $\ln \left(\mathrm{A}_{\mathrm{e}}-\mathrm{A}_{\mathrm{t}}\right) /\left(\mathrm{A}_{\mathrm{e}}-\mathrm{A}_{0}\right)$ is linearly related to time ( $\left.\mathrm{t}\right)$ from the previous report [47]. The slope of the curve is the self-assembly rate of the specimens. As mentioned earlier, the self-assembly of CMP can be divided into three stages. The initial nucleation stage and the nucleation growth stage determine its self-aggregation rate. Figure 4 shows the linear fitting graph of $\ln \left(\mathrm{A}_{\mathrm{e}}-\mathrm{A}_{\mathrm{t}}\right) /\left(\mathrm{A}_{\mathrm{e}}-\mathrm{A}_{0}\right)$ and $\mathrm{t}$ in the nucleation and growth stages of the CMP, GCD/ CMP, and GLH/CMP self-assembly process, respectively. The fitting self-aggregation rate constants of the twophase kinetic of CMP, GCD/CMP, and GLH/CMP are obtained from Fig. 4 by the least-squares method tabulated in Table 1. Table S1 exhibits the quantitative 


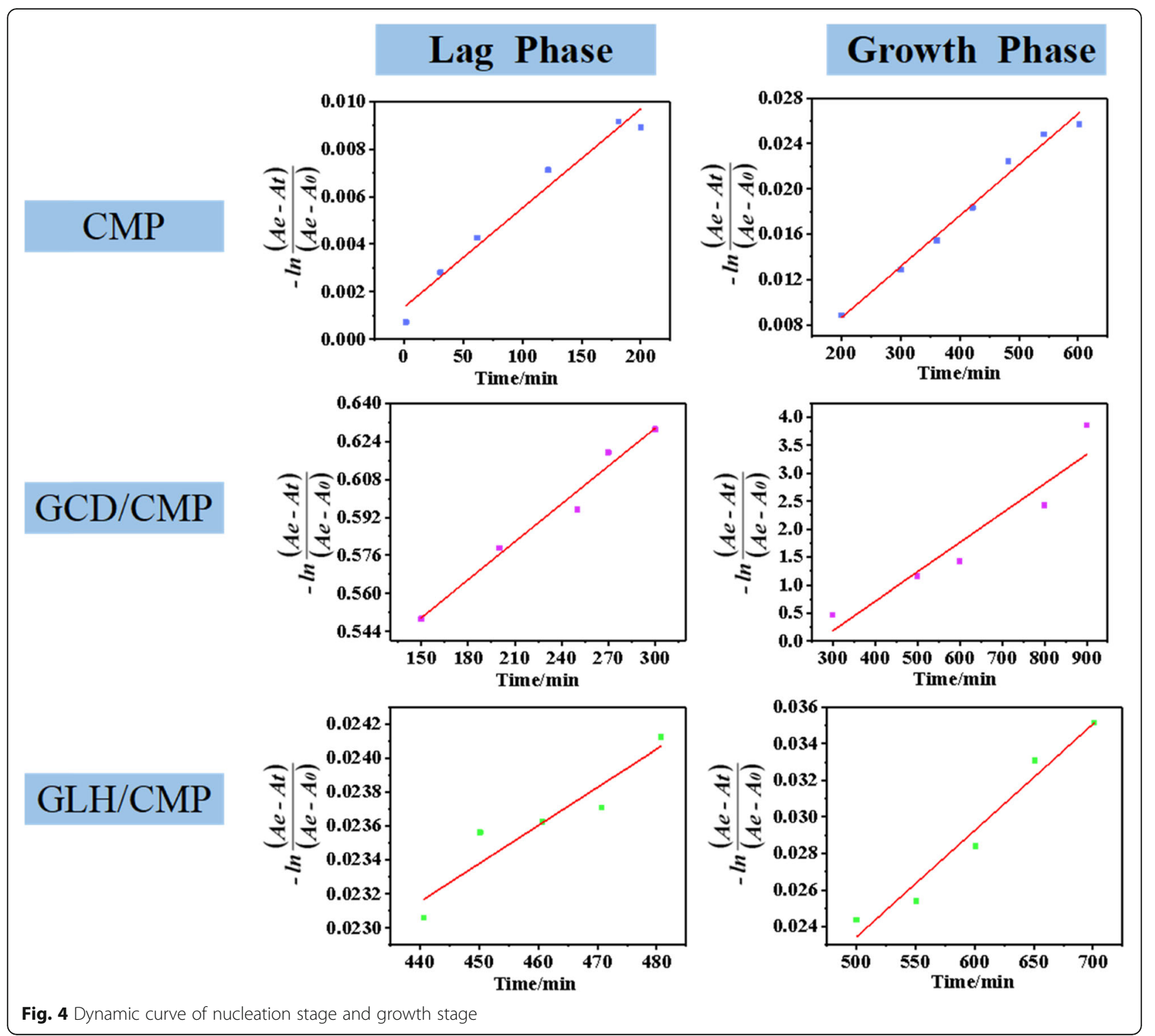

hydrophilic groups and hydrophobic groups of CMP in the presence of GCD and GLH, which substantiates that GCD/CMP and GLH/CMP have an increase of $9.3 \%$ of hydrophilic groups and $12.2 \%$ of hydrophobic groups compared with CMP, respectively. Figure 4 and Table 1 indicate that compared with CMP, the self-assembly rates of both GCD/CMP and GLH/CMP were reduced by about 3.3 times and 100 times in the initial nucleation

Table 1 Self-assembly rates of CMP, GCD/CMP and GLH/CMP

\begin{tabular}{lll}
\hline Sample & Lag phase $(\mathbf{L} /(\mathbf{g} \cdot \mathbf{c m} \cdot \mathbf{m i n}))$ & Growth phase $(\mathrm{L} /(\mathbf{g} \cdot \mathbf{c m} \cdot \mathbf{m i n}))$ \\
\hline $\mathrm{CMP}$ & $2.27 \times 10^{-3}$ & $1.46 \times 10^{-2}$ \\
$\mathrm{GCD} / \mathrm{CMP}$ & $5.32 \times 10^{-4}$ & $5.25 \times 10^{-3}$ \\
$\mathrm{GLH} / \mathrm{CMP}$ & $2.25 \times 10^{-5}$ & $5.82 \times 10^{-5}$ \\
\hline
\end{tabular}

stage, respectively, and decreased by about 2.8 times and 250 times in the growth stage, respectively. This maybe because the emerging hydrophobic and hydrophilic forces between CMP nuclei increases due to the incremental corresponding groups, thereby resulting in a prolonged time in the self-assembly kinetic process. Noted that whether in the nucleation growth stage or the initial nucleation stage, the assembly rate of GCD/CMP is two orders of magnitude faster than that of GLH/CMP, indicating that the hydrophilic effect in the growth stage is stronger driving effects than the hydrophobic force on molecular self-assembly under the experimental conditions. Consequently, both supernumerary hydrophilic groups and hydrophobic groups delay the self-assembly process of CMP owing to the altered hydrogen bonds 
and hydrophobic forces between CMP molecules, which is fortunately consistent with the above-mentioned turbidity analysis results.

\subsection{Performance analysis of CMP, GCD/CMP and GLH/CMP assemblies}

The hydrophilic and hydrophobic properties of CMP, GCD/CMP and GLH/CMP are evaluated by SCA. Figure $5 \mathrm{c}$ shows that the SCA of CMP and GCD/CMP are $52.8^{\circ}$ and $32.4^{\circ}$, respectively, exhibiting the hydrophilic nature. While, the SCA of GLH/CMP presents $140.7^{\circ}$, which is a highly hydrophobic substance. Figure $5 \mathrm{~b}$ displays the DCA of CMP, GCD/CMP and GLH/CMP. The DCA of CMP is finally stable at $40.4^{\circ}$, which shows that CMP has better hydrophilicity. For the GLH/CMP, it maintains at a higher DCA compared with CMP, indicating highly hydrophobic. This may be because the CMP has been hydrophobically modified to introduce hydrophobic Si and alkyl groups. For the GCD/CMP, the DCA quickly decreases to $0^{\circ}$ and remains at $0^{\circ}$, which could be speculated that it may be due to the reaction of the amino groups on the CMP molecular chain with the epoxy groups to generate more hydrophilic hydroxyl groups, indicating that the improved hydrophilic performance of CMP.

Figure $5 \mathrm{~d}$ exhibits the in vitro degradation properties of the CMP, GCD/CMP and GLH/CMP exposed to the SBF simulated body fluid. The results indicate that the CMP film is basically degraded after $5 \mathrm{~min}$. Homoplastically, the GCD/CMP film could be absolutely dissolved in the SBF solution in about $8 \mathrm{~s}$. Quite differently, the degradation rate of the GLH/CMP membrane are only $46 \%$ degraded after the immersion period of 15 days. Obviously, the introduction of hydrophobic groups could greatly improve the in vitro degradation resistance of CMP, which is significantly conducive to its biological applications.

\section{Conclusions}

The objective of this study is to evaluate the selfassembly mechanism of CMP, particularly the effect of hydrophilic and hydrophobic interactions between CMP bulk on its self-assembly behavior and morphology by grafting the hydrophilic GCD and hydrophobic GLH onto the side chains of CMP through the ring-opening reaction. The results exhibite that both the self-assembly process of the hydrophilic GCD/CMP and hydrophobic a

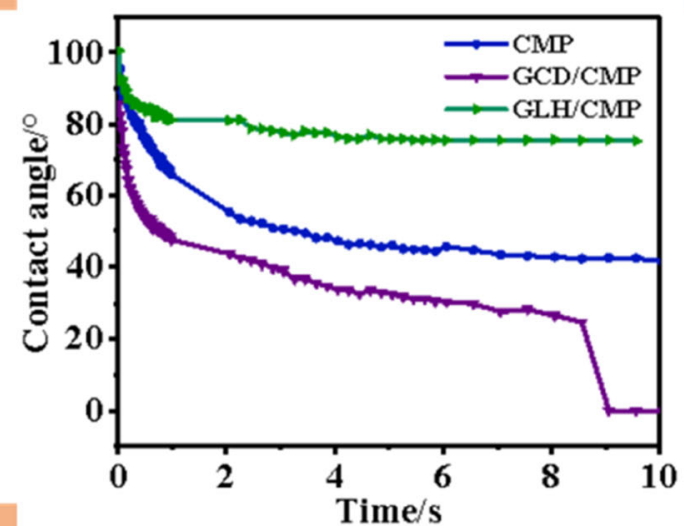

c

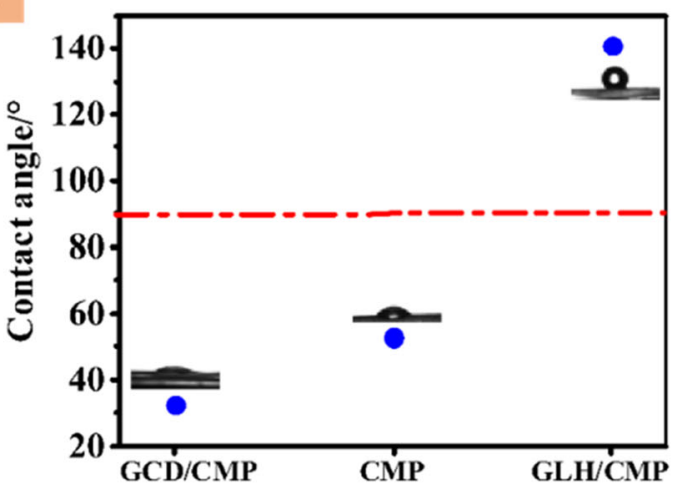

b

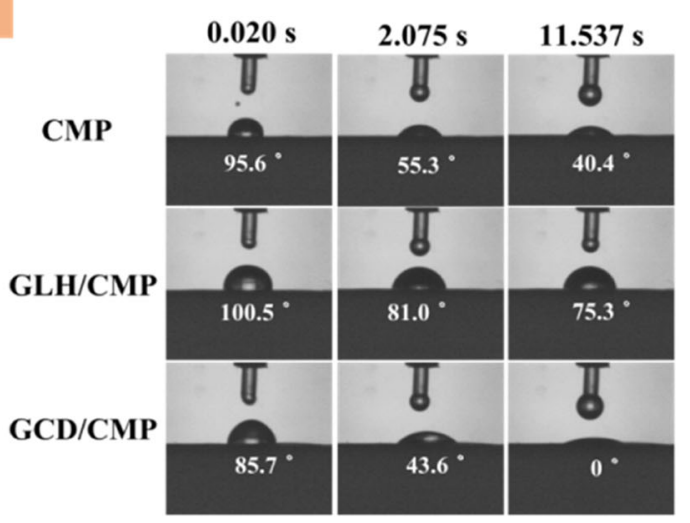

d

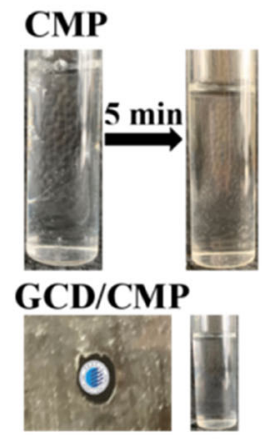

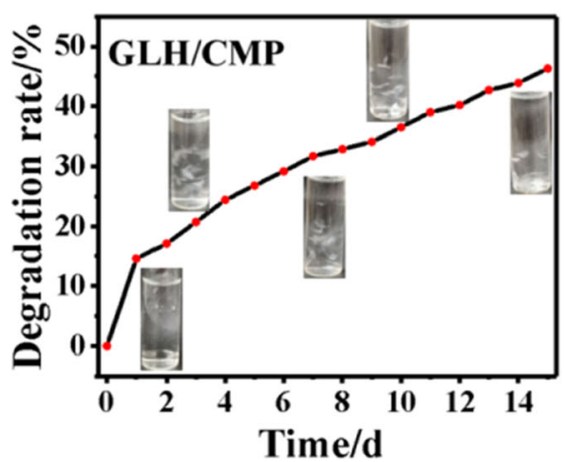

Fig. 5 a Contact Angle and time relationship; b The DCA diagram; c The SCA image; d Degradation of CMP, GCD/CMP and GLH/CMP 
GLH/CMP are stratified with the "nucleation-growth" mechanism. Meanwhile, the supernumerary hydrophilic or hydrophobic interactions between the CMP result in a prolonged self-ssembly period, and moderative reconstruction rates both in lag phase and growth phase, especially the nucleation growth period. Noted that, the selfassembly rate of the GCD/HGL is faster than that of the GLG/CMP in the nucleation growth stage, indicating that hydrophilic interactions might have stronger driving effects on CMP self-assembly in the experimental conditions. Moreover, we also confirm that the hydrophilic and hydrophobic interactions have a regulatory effect on the morphology and structure of CMP self-assembles. The hydrophilic forces promote CMP to generate nanofiberous structures, while hydrophobic interactions are more conducive to the formation ofthe microsphere structures. Besides, the GLH/CMP membrane has better resistance to degradation in vitro. This work for the first time reveals the influence of the hydrophilic and hydrophobic forces on the assembly process of CMP biomacromolecules, as well as the morphology and structure of self-assembles. Achieved precise control of the morphology of CMP assembles, and constructed a series of multi-scale molecular assembly functional systems, laying a theoretical and practical foundation for the application of CMP and its aggregates in drug delivery, wound healing, tissue engineering, and cell culture.

\begin{abstract}
Abbreviations
CMP: Collagen mimetic peptide; GCD: Glycidol; GLH: Y-glycidyl ether oxypropyl trimethoxysilan; SEM: Scanning electron microscope; SCA: Static contact angle; DCA: Dynamic contact angle; UV-vis: Ultraviolet-visible-nearinfrared spectrophotometer; FT-IR: Fourier Transform Infrared Spectroscopy; SBF: Simulating body fluids
\end{abstract}

\section{Supplementary Information}

The online version contains supplementary material available at https://doi. org/10.1186/s42825-021-00054-3.

Additional file 1 : Figure $\mathbf{S 1}$. The amino acid sequence of CMP. Figure S2. Amino conversion rates under different external conditions. Figure S3. The isoelectric point of of CMP, GCD/CMP and GLH/CMP. Figure S4. AFM diagram of HLC self-assembled microspheres for 600 min with microspheres size. Figure S5. AFM diagram of self-assembled HLC, where a, b, c and d are pH 5.0, 7.0, 8.0 and 9.0 respectively. Table S1. Hydrophilic and hydrophobic ratios.

\section{Acknowledgements}

Not applicable.

\section{Authors' contributions}

LXM was a major contributor in the conception of the study, the discussion of the results and the revision of the manuscript. $\mathrm{HQQ}$ performed the experiments about the hydrophilic and hydrophobic interaction and data analyses and writing the manuscript. ZC and LY performed the experiments about morphology determination and data analyses. LXH performed the revision of the manuscript, and was a major contributor in data analyses and writing the manuscript. All authors read and approved the final manuscript.

Authors' information

Not applicable.

\section{Funding}

This work was financially supported by the National Natural Science Foundation of China (21808133), Scientific Research Foundation for Young Scholars of Shaanxi University of Science \& Technology (contract grant number 2017BT-32), Xianyang Science and Technology Project (Y20190138), and Shaanxi Province Key R\&D Program (2018ZDXM-SF-091).

\section{Availability of data and materials}

All data needed to evaluate the conclusions in the paper are presented in the paper and the supplementary materials.

\section{Declaration}

\section{Competing interests}

The authors declare no competing interest.

\section{Author details}

${ }^{1}$ College of Bioresources Chemical and Materials Engineering, Shaanxi University of Science \& Technology, Wei Yang District, Xi'an 710021, Shaanxi, China. ${ }^{2}$ National Demonstration Center for Experimental Light Chemistry Engineering Education, Shaanxi University of Science \& Technology, Weiyang District, Xi'an 710021, Shaanxi, China. ${ }^{3}$ Institute of Biomass \& Functional Materials, Shaanxi University of Science \& Technology, Weiyang District, Xi'an 710021, Shaanxi, China.

Received: 9 December 2020 Accepted: 2 March 2021

Published online: 15 April 2021

\section{References}

1. Okesola BO, Mata A. Multicomponent self-assembly as a tool to harness new properties from peptides and proteins in material design. Chem Soc Rev. 2018;47(10):3721-36. https://doi.org/10.1039/c8cs00121a.

2. Ulijn RV, Jerala R. Peptide and protein nanotechnology into the 2020s: beyond biology. Chem Soc Rev. 2018;47(10):3391-4. https://doi.org/10.1039/ c8cs90055h

3. Raymond DM, Nilsson BL. Multicomponent peptide assemblies. Chem Soc Rev. 2018:47(10):3659-720. https://doi.org/10.1039/C8CS00115D.

4. Hong LY, Leng LC, Wenxiong $S$, et al. Creating two self-assembly microenvironments to achieve supercrystals with dual structures using polyhedral nanoparticles. Nat Commun. 2018;9(1):2769. https://doi.org/10.1038/s41467018-05102-x.

5. Mclaughlin S, Mcneill B, Podrebarac J, et al. Injectable human recombinant collagen matrices limit adverse remodeling and improve cardiac function after myocardial infarction. Nat Commun. 2019;10(1):4866. https://doi.org/1 0.1038/s41467-019-12748-8.

6. Tytgat L, Markovic M, Qazi TH, et al. Photo-crosslinkable recombinant collagen mimics for tissue engineering applications. J Mater Chem B. 2019; 7(19):3100-8. https://doi.org/10.1039/c8tb03308k.

7. Chi L, Fan D, Ma X, et al. A genetic algorithm for the optimization of the thermoinduction protocol for high-level production of recombinant humanlike collagen from Escherichia coli. Biotechnol Appl Biochem. 2011;58(3): 175-84. https://doi.org/10.1002/bab.25.

8. Liu Y, Gu J, Fan D. Fabrication of high-strength and porous hybrid scaffolds based on nano-hydroxyapatite and human-like collagen for bone tissue regeneration. Polymers. 2020;12(1):61. https://doi.org/10.3390/polym1201 0061.

9. Yan J, Hu K, Xiao Y, et al. Preparation of recombinant human-like collagen/ fibroin scaffold and its promoting effect on vascular cells biocompatibility. J Bioact Compat Polym. 2018;33(4):416-25. https://doi.org/10.1177/088391151 8769680.

10. Yuan M, Dai F, Li D, et al. Lysozyme/collagen multilayers layer-by-layer deposited nanofibers with enhanced biocompatibility and antibacterial activity. Mater Sci Eng C. 2020;112(0928-4931):110868. https://doi.org/10.101 6/j.msec.2020.110868.

11. Song $X$, Zhu C, Fan D, et al. Erratum: a novel human-like collagen hydrogel scaffold with porous structure and sponge-like properties. Polymers. 2018; 10(3):638. https://doi.org/10.3390/polym9120638.

12. Tu H, Wu G, Yi Y, et al. Layer-by-layer immobilization of amphoteric carboxymethyl chitosan onto biocompatible silk fibroin nanofibrous mats. Carbohydr Polym. 2019;210(0144-8617):9-16. https://doi.org/10.1016/j.ca rbpol.2019.01.047. 
13. Yang W, Meyers MA, Ritchie RO. Structural architectures with toughening mechanisms in nature: a review of the materials science of typecollagenous materials. Prog Mater Sci. 2019;103(6):425-83. https://doi.org/1 0.1016/j.pmatsci.2019.01.002

14. Xu Z, Chang J, Zhang P, et al. Collagen modified with epoxidized safrole for improving antibacterial activity. RSC Adv. 2017;7(79):50300-6. https://doi. org/10.1039/c7ra08319j.

15. Cheng D, Zhang X, Gao Y, et al. Endogenous reactive oxygen speciestriggered morphology transformation for enhanced cooperative interaction with mitochondria. J Am Chem Soc. 2019;141(18):7235-9. https://doi.org/1 $0.1021 / j a c s .8 b 07727$.

16. Fenyves R, Schmutz M, Horner IJ, et al. Aqueous self-assembly of Giant bottlebrush block copolymer surfactants as shape-tunable building blocks. J Am Chem Soc. 2014;136(21):7762-70. https://doi.org/10.1021/ja503283r.

17. Li P, Sun M, Xu Z, et al. Site-selective in situ growth-induced self-assembly of protein-polymer conjugates into $\mathrm{pH}$-responsive micelles for tumor microenvironment triggered fluorescence imaging. Biomacromolecules. 2018;19(11):4472-9. https://doi.org/10.1021/acs.biomac.8b01368.

18. Guo W, Zhang Z, Wei Q, et al. Intracellular restructured reduced glutathione-responsive peptide Nanofibers for synergetic tumor chemotherapy. Biomacromolecules. 2020;21(2):444-53. https://doi.org/10.1 021/acs.biomac.9b01202

19. Lu Y, Aimetti AA, Langer R, et al. Bioresponsive materials. Nat Rev Mater. 2016;1(1):16075. https://doi.org/10.1038/natrevmats.2016.75.

20. Yang G, Ding H-m, Kochovski Z, et al. Highly ordered self-assembly of native proteins into 1D, 2D and 3D structures modulated by a tether length of inducing ligands. Angew Chem. 2017;129(36). https://doi.org/10.1002/anie.2 01703052

21. Yan J, He W, Yan S, et al. Self-assembled peptide-lanthanide nanoclusters for safe tumor therapy: overcoming and utilizing biological barriers to peptide drug delivery. ACS Nano. 2018;12(2):2017-26. https://doi.org/10.1 021/acsnano.8b00081.

22. Engelberg Y, Landau M. The human LL-37(17-29) antimicrobial peptide reveals a functional supramolecular structure. Nat Commun. 2020;11(1):3894. https://doi.org/10.1038/s41467-020-17736-X.

23. Li $N$, Zhang $M$, Zha $Y$, et al. $\pi-\pi$ stacking-directed self-assembly of nanoplatelets into diversified three-dimensional superparticles for high surface-enhanced Raman scattering. J Colloid Interface Sci. 2020;575(00219797):54-60. https://doi.org/10.1016/j.jcis.2020.04.088.

24. Bruel C, Davies TS, Carreau PJ, et al. Self-assembly behaviors of colloidal cellulose nanocrystals: a tale of stabilization mechanisms. J Colloid Interface Sci. 2020:574(0021-9797):399-409. https://doi.org/10.1016/j.jcis.2020.04.049.

25. Wang Y, Li G, Zhang Y, Li L, Shang M. Layer-by-layer assembly into bulk-like $\mathrm{g}-\mathrm{C} 3 \mathrm{~N} 4$ via artificial manipulation of electrostatic forces. Chem Commun. 2020:56(100):15663-6. https://doi.org/10.1039/D0CC05851C.

26. Olga AK, Artem VN, Alexander FS, et al. Water-soluble fullerene-based nanostructures with promising antiviral and myogenic activity. Chem Commun. 2020;56(17):10203-6. https://doi.org/10.1039/d0cc03928d.

27. Dhamecha D, Le D, Movsas R, et al. Porous polymeric microspheres with controllable pore diameters for tissue engineered lung tumor model development. Front Bioeng Biotechnol. 2020;8:799. https://doi.org/10.3389/ fbioe.2020.00799.

28. Yang $X$, Wang $X$, Hong $H$, et al. Galactosylated chitosan-modified ethosomes combined with silk fibroin nanofibers is useful in transcutaneous immunization. J Control Release. 2020:327(0168-3659):88-99. https://doi. org/10.1016/j.jconrel.2020.07.047.

29. Kontturi $E$, Laaksonen $P$, Linder MB, et al. Advanced materials through assembly of Nanocelluloses. Adv Mater. 2018;30(24):1703779. https://doi. org/10.1002/chem.202003269.

30. Sun $Y$, Shieh A, Kim SH, et al. The self-assembly of a camptothecin-lysine nanotube. Bioorg Med Chem Lett. 2016;26(12):2834-8. https://doi.org/10.101 6/j.bmcl.2016.04.056

31. Kobayashi $Y$, Arai N. Self-assembly of surfactant aqueous solution confined in a Janus amphiphilic nanotube. Mol Simul. 2017:43(13-16):1153-9. https:// doi.org/10.1080/08927022.2017.1319060

32. Sun $Y$, Fry $C M$, Shieh $A$, et al. Self-assembly of a robust, reduction-sensitive camptothecin nanotube. Chem Commun. 2020;56(71):10333-6. https://doi. org/10.1039/D0CC04396F.

33. $X u Y$, Huang $Y$, Zhang $X$, et al. Carrier-free Janus nano-prodrug based on camptothecin and gemcitabine: reduction-triggered drug release and synergistic in vitro antiproliferative effect in multiple cancer cells. Int Pharm. 2018;550(1-2):45-56. https://doi.org/10.1016/j.ijpharm.2018.08.041.

34. He W, Du Y, Zhou W, et al. Core-crosslinked nanomicelles based on crosslinkable prodrug and surfactants for reduction responsive delivery of camptothecin and improved anticancer efficacy. Eur J Pharm Sci. 2020; 150(0928-0987):105340. https://doi.org/10.1016/j.ejps.2020.105340.

35. Oun AA, Shankar S, Rhim J. Multifunctional nanocellulose/metal and metal oxide nanoparticle hybrid nanomaterials. Crit Rev Food Sci Nutr. 2020;60(3): 435-60. https://doi.org/10.1080/10408398.2018.1536966.

36. Wagberg L, Erlandsson J. The use of layer-by-layer self-assembly and nanocellulose to prepare advanced functional materials. Adv-Mater. 2020: e2001474. https://doi.org/10.1002/adma.202001474.

37. Shi W, Yang Y, Lee HK, et al. Modulating orientational order to organize polyhedral nanoparticles into plastic crystals and uniform metacrystals. Angew Chem Int Ed. 2020;59(47):21183-21189(7). https://doi.org/10.1002/a nie.202009941.

38. Dommelen RV, Fanzio P, Sasso L. Surface self-assembly of colloidal crystals for micro- and nano-patterning. Adv Colloid Interf Sci. 2018;251(0001-8686): 97-114. https://doi.org/10.1016/j.cis.2017.10.007.

39. Liao H, Lin J, Liu Y, et al. Self-assembly mechanisms of nanofibers from peptide amphiphiles in solution and on substrate surfaces. Nanoscale. 2016; 8(31):14814-20. https://doi.org/10.1039/C6NR04672J.

40. Rey M, Yu T, Bley K, et al. Amphiphile-induced anisotropic colloidal selfassembly. Langmuir. 2018;34(34):9990-10000. https://doi.org/10.1021/acs.la ngmuir.8b01382.

41. Rey M, Law AD, Buzza DMA, et al. Anisotropic self-assembly from isotropic colloidal building blocks. J Am Chem Soc. 2017;139(48):17464-73. https:// doi.org/10.1021/jacs.7b08503.

42. Fonseca ER, Mendoza Cl. Self-assembly of core-corona particles confined in a circular box. J Phys Condens Matter. 2020;32(1):15101. https://doi.org/10.1 088/1361-648X/ab42fc

43. Liu H, Lin M, Liu X, et al. Doping bioactive elements into a collagen scaffold based on synchronous self-assembly/mineralization for bone tissue engineering. Bioactive Mater. 2020;5(4):844-58. https://doi.org/10.1016/j.bioa ctmat.2020.06.005.

44. Rey M, Fernandez-Rodriguez MA, Karg M, et al. Poly-N-isopropylacrylamide nanogels and microgels at fluid interfaces. Acc Chem Res. 2020:53(2):41424. https://doi.org/10.1021/acs.accounts.9b00528.

45. Liu X, Zheng C, Luo X, et al. Recent advances of collagen-based biomaterials: Multi-hierarchical structure, modification and biomedical applications. Materi Sci Eng : C. 2019;99(0928-4931):1509-22. https://doi. org/10.1016/j.msec.2019.02.070

46. Tsai SW, Liu RL, Hsu FY, et al. A study of the influence of polysaccharides on collagen self-assembly: nanostructure and kinetics. Biopolymers. 2006;83(4): 381-8. https://doi.org/10.1002/bip.20568.

47. Guo $Y, X u B$, Wang $Y$, et al. Dramatic promotion of wound healing using a recombinant human-like collagen and bFGF cross-linked hydrogel by transglutaminase. J Biomater Sci Polym Ed. 2019;30(17):1591-603. https:// doi.org/10.1080/09205063.2019.1652416.

48. Zhang $Y$, Zhang $Y$, Cui $H$, et al. Dynameric collagen self-healing membranes with high mechanical strength for effective cell grow applications. Chem A Eur J. 2020;26(71):16994-16999(6). https://doi.org/10.1002/chem.202003269.

\section{Publisher's Note}

Springer Nature remains neutral with regard to jurisdictional claims in published maps and institutional affiliations. 